\title{
Agreement of a Short Form of the Self-Administered Multidimensional Prognostic Index (SELFY-MPI-SF): A Useful Tool for the Self-Assessment of Frailty in Community-Dwelling Older People
}

This article was published in the following Dove Press journal:

Clinical Interventions in Aging

\begin{abstract}
Alberto Cella'
Alberto Ferrari $\left(\mathbb{D}^{2}\right.$

Giuseppe Rengo (iD ${ }^{3}$

Vincenzo Solfrizzi ${ }^{4}$

Nicola Veronese ${ }^{5}$

Matteo Puntoni ${ }^{6}$

Sabrina Zora'

Alberto Pilotto ${ }^{1,4}$

Filippo Fimognari ${ }^{7}$
\end{abstract}

On behalf of the SELFY-MPI

SIGOT Investigators

'Geriatric Unit, Department of Geriatric Care, Orthogeriatrics and Rehabilitation, EO Galliera Hospital, Genova, Italy;

${ }^{2}$ Geriatric Unit, Arcispedale S. Maria Nuova, AUSL Reggio Emilia, Reggio

Emilia, Italy; ${ }^{3}$ Department of Translational Medical Sciences, Division of Geriatrics,

Federico II University, Napoli, Italy;

${ }^{4}$ Department of Interdisciplinary

Medicine, University of Bari, Bari, Italy;

${ }^{5}$ Primary Care Department, Azienda

ULSS 3 Serenissima, Venice, Italy;

${ }^{6}$ Scientific Coordination Unit, EO

Galliera Hospital, Genova, Italy;

${ }^{7}$ Geriatric Unit, Department of Internal

Medicine, Azienda Ospedaliera di

Cosenza, Cosenza, Italy

Correspondence: Sabrina Zora Geriatric Unit, Department of Geriatric Care, Orthogeriatrics and Rehabilitation, EO Galliera Hospital, Genova, Italy Email sabrina.zora@galliera.it
Background: The Multidimensional Prognostic Index (MPI) is a comprehensive geriatric assessment (CGA)-based tool that has shown excellent accuracy in predicting negative health outcomes in older people. Recently, the self-administered version of MPI (SELFYMPI) has been validated in a community-dwelling sample, revealing excellent agreement with the original MPI. In the SELFY-MPI, Gijon's social-familial evaluation scale (SFES) was used to assess socio-relational and economic aspects. Completion of the SELFY-MPI, however, requires a significant amount of time in people aged over 60 years, particularly to fill in the SFES scale. The aim of this study was to validate, in a sample of community-dwelling older people, a short-form version of the SELFY-MPI (SELFY-MPI-SF), in which the SFES scale was replaced by the "co-habitation status" domain, as in the original version of the MPI.

Methods: All participants included in the study completed both versions of the selfadministered MPI, which share the following seven domains: 1) basic and 2) instrumental activities of daily living, 3) mobility, 4) cognition, 5) nutrition, 6) comorbidity, and 7) number of medications. Moreover, in the SELFY-MPI-SF, the 8th domain "co-habitation status" (ie living alone, with family or in a residential facility) replaced the SFES scale. The Bland-Altman methodology was applied in order to measure the agreement between the two instruments. Finally, the time to complete the SFES scale and the question on co-habitation was measured.

Results: The final study sample was composed of 129 participants (mean age $=76.8$ years, range $=65-93$ years, $64.3 \%$ women) were enrolled. The mean SELFY-MPI and SELFY-MPISF values were $0.221 \pm 0.196$ and $0.246 \pm 0.188$, respectively. The mean difference was clinically irrelevant $(-0.025 \pm 0.058)$. None of the 129 observations showed values outside the established $5 \%$ limits of agreement. The agreement between SELFY-MPI and SELFYMPI-SF was excellent $(\mathrm{k}=0.762$; $\mathrm{rho}=0.924, \mathrm{p}<0.0001$ for both). Stratified analyses of agreement among subgroups of participants of different ages did not show any significant differences between the two versions. Completion of the SFES required about 7 mins, on average, while the question on habitation status required about $10 \mathrm{~s}$.

Conclusion: The SELFY-MPI-SF showed strong agreement and precision when compared with the standard SELFY-MPI in people aged 65 and older and can therefore be successfully used as a quicker self-administered frailty instrument in community-dwelling older people.

Keywords: comprehensive geriatric assessment, multidimensional prognostic index, frailty, self assessment 


\section{Introduction}

Prognostic tools have increasingly been used in current geriatric medicine in order to improve clinical decisionmaking in diagnostics and therapeutics, and also to identify frail older people in order to tailor appropriate interventions. Multimorbidity and domains such as physical and cognitive function and biological and social determinants are key factors in formulating the prognosis of older people. ${ }^{1}$ Comprehensive Geriatric Assessment (CGA) has been recognized as a multidimensional, interdisciplinary diagnostic process able to effectively explore all these multiple domains of health, and is widely used to determine the clinical profile, pathological risk, residual skills, and short- and long-term prognosis in frail older people. $^{2}$

Among the few mortality prognostic indices used in geriatric medicine, the Multidimensional Prognostic Index $(\mathrm{MPI})^{3}$ has proved to be a well-calibrated tool with good discrimination and accuracy in predicting short- and long-term mortality in both hospital ${ }^{4}$ and community settings. ${ }^{5}$ The MPI is a CGA-based prognostic tool that collects information on functional and cognitive aspects, mobility, nutrition, polypharmacy, comorbidity and cohabitation status through standardized and validated rating scales. ${ }^{3}$

The ability of MPI to identify short- and long-term mortality and other negative health outcomes has been demonstrated by several multicenter studies, making this instrument a valuable aid to the clinical decision-making process in multimorbid older patients ${ }^{6}$ and one of the reference tools for assessing frailty. ${ }^{7,8}$ According to the growing interest in self-perception of health status in current medicine, a self-administered version of a clinical assessment tool has been developed. Hence, the selfperception of frailty could allow an early diagnosis of this syndrome. Nevertheless, a recent review underlined that data on self-reported tools regarding frailty assessment in older people were limited ${ }^{9}$ and that only physical frailty was the dimension that could be assessed on the basis of the criteria proposed by Fried et al. ${ }^{10}$

Very recently, a self-administered version of MPI (SELFY-MPI) was developed and validated in a community-dwelling population within the framework of the European Union co-funded project EFFICHRONIC. ${ }^{11}$ The SELFY-MPI showed similar values to the standard MPI, and their agreement proved to be age-independent. ${ }^{11}$ Moreover, preliminary data on this easy-to-use tool indicate that it has been implemented in five European countries, attesting to its feasibility in different national contexts and in heterogeneous populations. ${ }^{12}$

However, even though the SELFY-MPI is a quick and easy tool, it has shown a significant difference in filling time between people aged under 60 years and those over 60 years: in the younger age-group, the mean value was 13.5 mins (standard deviation \pm 4.7 ), while in older participants the mean value was 21.7 mins (standard deviation \pm 9.2 ). ${ }^{12}$ A significant portion of the completion time is required by Gijon's social-familiar evaluation scale (SFES), which was used in the EFFICHRONIC project for the in-depth assessment of social marginalization in a sample of adults of all ages. ${ }^{12}$ Although an in-depth assessment of the social dimension could be particularly relevant for older people, information on cohabitation status, and particularly the condition of living alone, is also relevant in assessing social determinants of frailty. Living alone is, in itself, associated with a higher risk of unplanned hospitalization, ${ }^{13}$ a higher risk of falling, higher levels of disease and disability, ${ }^{14}$ and even a higher risk of mortality. ${ }^{15}$

We therefore constructed a short-form version of the SELFY-MPI (SELFY-MPI-SF) in order, on the one hand, to place greater emphasis on the loneliness of older people as a potential risk factor, and mainly to facilitate selfadministration of the test by older people by shortening its completion time.

The aim of the present study was to compare the precision and agreement of this new short-form version of the SELFY-MPI with the previously validated SELFYMPI. ${ }^{11}$ The only difference between the two instruments is that the SFES (included in the standard version of the SELFY-MPI) has been replaced by the question on cohabitation status in the SELFY-MPI-SF.

\section{Methods}

\section{Study Design and Population}

This observational study was conducted between January and July 2019 in accordance with the World Medical Association's 2008 Declaration of Helsinki, the guidelines for Good Clinical Practice, and the Strengthening the Reporting of Observational Studies in Epidemiology (STROBE) guidelines. ${ }^{16}$

The inclusion criteria were: (1) age 65 years or over; (2) community-dwelling people who attended the outpatient clinics of Galliera Hospital; (3) absence of acute clinical conditions; (4) ability to provide informed consent. 
All participants read and signed the informed consent form and all participants' records and personal information were rendered anonymous before statistical analysis.

The Ethics Committee of the Liguria Region (Italy) approved this study.

\section{Self-Administered MPI (Standard SELFY-MPI)}

The standard SELFY-MPI ${ }^{11}$ considers the following eight domains, assessed through eight self-administered scales (Table S1):

1. Functional status, measured by the Barthel ADL scale, ${ }^{17}$ which assesses feeding, bathing, personal hygiene, dressing, fecal and urinary continence and toilet use. This scale can be self-assessed. ${ }^{18}$

2. Mobility, assessed through the Barthel mobility ${ }^{17}$ scale, which measures the ability to get in and out of bed/chair, walk and go up and down the stairs. This scale can also be self-administered. ${ }^{18,19}$

3. Independence in the instrumental activities of daily living, ie, telephone use, grocery shopping, meal preparation, housekeeping, laundry, travel, medication, handling finances; these items are assessed through the self-administered version of Lawton's Instrumental Activities of Daily Living (IADL) scale. ${ }^{20}$

4. Cognitive status, investigated by means of the selfadministered cognitive screening test, Test Your Memory (TYM). ${ }^{21}$ This 10 -task cognitive test explores several domains, such as orientation, ability to copy a sentence, semantic knowledge, calculation, verbal fluency, similarities, naming, visuo-spatial abilities and recall of a previously copied sentence. The score ranges from 0 to 50 , higher scores indicating better cognitive function. ${ }^{21}$

5. Nutritional status, measured by means of the Mini Nutritional Assessment Short Form (MNA-SF) ${ }^{22}$ through its validated self-administered version. ${ }^{23}$ This scale is composed of 6 items and includes information on (1) decline in food intake, (2) weight loss, (3) mobility, (4) recent psychological stress, (5) neuropsychological problems and (6) anthropometric measures (body mass index and weight loss).

6. The number of medications regularly taken by the subject.

7. Comorbidity, evaluated by means of the Cumulative Illness Rating Scale (CIRS), ${ }^{24}$ which explores the number of chronic health diseases requiring drug therapies in 13 health areas: (1) cardiac, (2) hypertension, (3) vascular, (4) respiratory, (5) eye, ear, nose, throat, larynx, (6) upper gastrointestinal (esophagus, stomach, pancreas), (7) lower gastrointestinal (intestines, hernias), (8) hepatic and pancreatic, (9) renal, (10) genitourinary (ureters, bladder, urethra, prostate, genitals) or gynecological, (11) muscles, bone and skin, (12) neurological (stroke, multiple sclerosis), (13) endocrine-metabolic (diabetes, thyroid, high cholesterol). Self-assessment of this scale is possible since participants are asked to report health problems for which they receive pharmacological treatment.

8. Socioeconomic domain, explored through the adapted version of the self-administered Gijon's social-familial evaluation scale (SFES). ${ }^{25}$ This scale assesses the subject's household composition, net monthly household income, living accommodation, social relationships and social support received. The SFES is a self-administered scale composed of 5 items, the maximum score being 25 points. Higher scores indicate a major social risk: scores between 10 and 14 indicate a social risk, and scores over 15 social problems. ${ }^{26}$

For each domain, a tripartite hierarchy is used, whereby a score of 0 indicates no problems, 0.5 minor problems and 1.0 major problems. This scoring is based on the conventional literature on each scale. The sum of these eight domains has to be divided by 8 in order to obtain a final SELFY-MPI risk score, ranging from 0 (= no risk) to 1 (= high risk), using the tripartite hierarchy in $0-0.33$ (low risk of mortality), 0.34-0.66 (moderate risk) and 0.67-1.0 (severe risk), according to the traditional division of this score. ${ }^{11}$

\section{SELFY-MPI Short-Form}

The SELFY-MPI-SF includes the first seven items of the standard SELFY-MPI (Table S1), ie, Barthel ADL, Barthel MOB, IADL, TYM test, MNA-SF, number of medications, and CIRS. In the eighth domain, however, the SFES scale is replaced by a tripartite item that includes three different living conditions: living alone, living in a residential facility, living with family members (see Table S2). Different levels of risk are attributed to each condition; living alone is considered a high-risk condition, living in a residential facility a moderate-risk condition 
and living with family members (such as spouse, offspring, relatives) a low-risk condition.

The same tripartite hierarchy as the standard SELFYMPI is used and the same scoring is applied in order to obtain a final SELFY-MPI-SF risk score ranging from 0 , low risk, to 1 , higher risk of negative outcomes.

\section{Statistical Analysis}

The main descriptive statistics were: absolute and relative (\%) frequencies; mean; standard deviation (SD); median; and minimum, maximum, and interquartile range (IQR). Mean differences were tested by means of the paired sample $t$-test.

The relationship between SELFY-MPI and SELFYMPI-SF was assessed through several methods. First, the agreement between these two measures, divided into categories, was estimated using the kappa coefficient; values less than 0.4 can be considered as poor, from 0.4 to 0.75 fair to good, and over 0.75 excellent. ${ }^{27}$ The agreement between the two tools was assessed as continuous measures using the Spearman's rank correlation coefficient (rho), due to the non-normal distribution of the two variables of interest: values close to zero indicate a low association between variables and those close to -1 or +1 indicate a strong linear association between two variables. Finally, precision was assessed using the Bland-Altman plots. Although scatter plots are an excellent way of checking the correlation between two outcomes, this method has low sensitivity in assessing the agreement between two measures. The Bland-Altman plot (BAP) ${ }^{28}$ methodology was used, since it provides an objective measure (95\% limits of agreement) and a visual representation (plot of the difference vs the mean of the two measures) of the level of agreement between two different continuous measures. In order to detect possible agedependent differences in the level of agreement, a BAP analysis stratified by deciles of age was performed.

We report two-tailed probabilities; a p-value of 0.05 was adopted to define nominal statistical significance. All analyses were conducted by means of the STATA software (version 14.2; StataCorp., Station, TX).

\section{Results}

A total of 129 participants completed both versions of the SELFY-MPI questionnaire; their mean age was 76.8 years (standard deviation, $\mathrm{SD} \pm 6.16$ years; range $65-93$ years) and most were women (64.3\%) (Table 1). In this cohort, mean standard SELFY-MPI and SELFY-MPI-SF values were $0.221(\mathrm{SD} \pm 0.196)$ and $0.246(\mathrm{SD} \pm 0.188)$, respectively.
Table I Participants' Characteristics

\begin{tabular}{|l|l|}
\hline Age, Years & \\
Mean (SD) & $76.8(6.2)$ \\
Median (IQR) & $78(72-8 I)$ \\
Min/max & $65 / 93$ \\
\hline Gender, n (\%) & \\
Men & $46(35.6)$ \\
Women & $83(64.3)$ \\
\hline Diseases, $n$ (\%) & \\
Hypertension & $77(59.7)$ \\
Cardiac & $32(24.8)$ \\
Vascular & $25(19.4)$ \\
Musculo-Skeletal & $25(19.4)$ \\
Endocrine-Metabolic & $25(19.4)$ \\
UPper GI & $23(17.9)$ \\
Lower GI & $10(7.8)$ \\
Genitourinary & $20(15.5)$ \\
ENT & $15(11.6)$ \\
Neurological & $8(6.2)$ \\
Respiratory & $7(5.4)$ \\
Liver & $2(1.6)$ \\
Kidney & $1(0.8)$ \\
\hline Standard SELFYMPI & \\
Mean (SD) & $0.0(-0.063-0.0)$ \\
Median (IQR) & $-0.125 / 0.063$ \\
\hline Min/max & $0.221(0.196)$ \\
\hline MeLFY-MPI-SF & 0.188 \\
Mean (SD) & $(0.063-0.250)$ \\
Median (IQR) & $0.0 / 0.813$ \\
\hline Mifference (SELFY-MPI-SF; Standard SELFY- & \\
MPI) (SD) & $0.0 / 0.813$ \\
\hline
\end{tabular}

Abbreviations: IQR, interquartile range; SD, standard deviation; Gl, gastrointestinal; ENT, eye, nose, throat, larynx.

The mean difference between the standard SELFY-MPI and its short-form version (SELFY-MPI-SF) was -0.025 (SD \pm $0.058)(p=0.316)$. The agreement between SELFY-MPI and SELFY-MPI-SF was excellent using both the kappa coefficient $(\mathrm{k}=0.762, \mathrm{p}<0.0001)$ and the rho coefficient $(\mathrm{rho}=0.924$, $\mathrm{p}<0.0001)$.

Hypertension was the most common disease, being reported by $77 / 129$ participants (59.7\%), followed by cardiac diseases in $32(24.8 \%)$, vascular, musculo-skeletal and endocrine-metabolic diseases in 25 participants each 


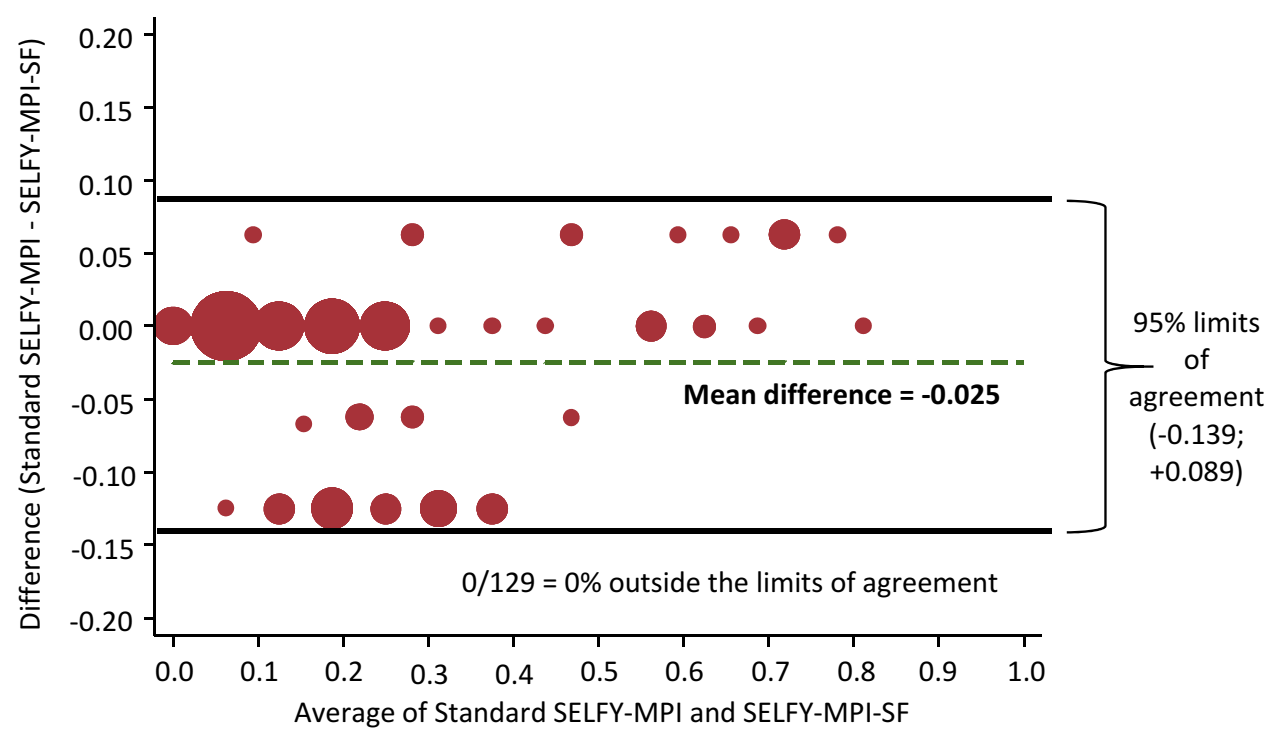

Figure I Bap of agreement between standard SELFY-MPI and SELFY-MPI-SF.

(19.4\%), gastroenteric conditions (upper and lower) in 33 $(25.6 \%)$ and genitourinary diseases in $20(15.5 \%)$.

Figure 1 shows the BAPs of the association between the two SELFY-MPI versions: none of the 129 participants included in the study showed values outside the limits of agreement.

A stratified analysis of agreement among subgroups of participants subdivided by deciles of age was performed. As shown in Figure 2, across the deciles of age the difference between the standard SELFY-MPI and its short-form version (SELFY-MPI-SF) was not statistically significant. Moreover, within each age-category, no significant differences between the two versions of SELFY-MPI emerged.
With regard to completion time, we observed a highly significant $(\mathrm{p}<0.001)$ statistical difference between the time needed to complete the SFES scale (about 7 mins) and the time needed to complete the question on cohabitation status (about $10 \mathrm{~s}$ ) (Table S3).

\section{Discussion}

A self-administered tool able to evaluate frailty is currently a target for welfare and health-care systems, since the self-perception of frailty could yield a wider assessment and enable frailty to be diagnosed earlier. ${ }^{29}$ So far, the self-assessment of frailty has been used only for screening purposes. Moreover, a systematic review found

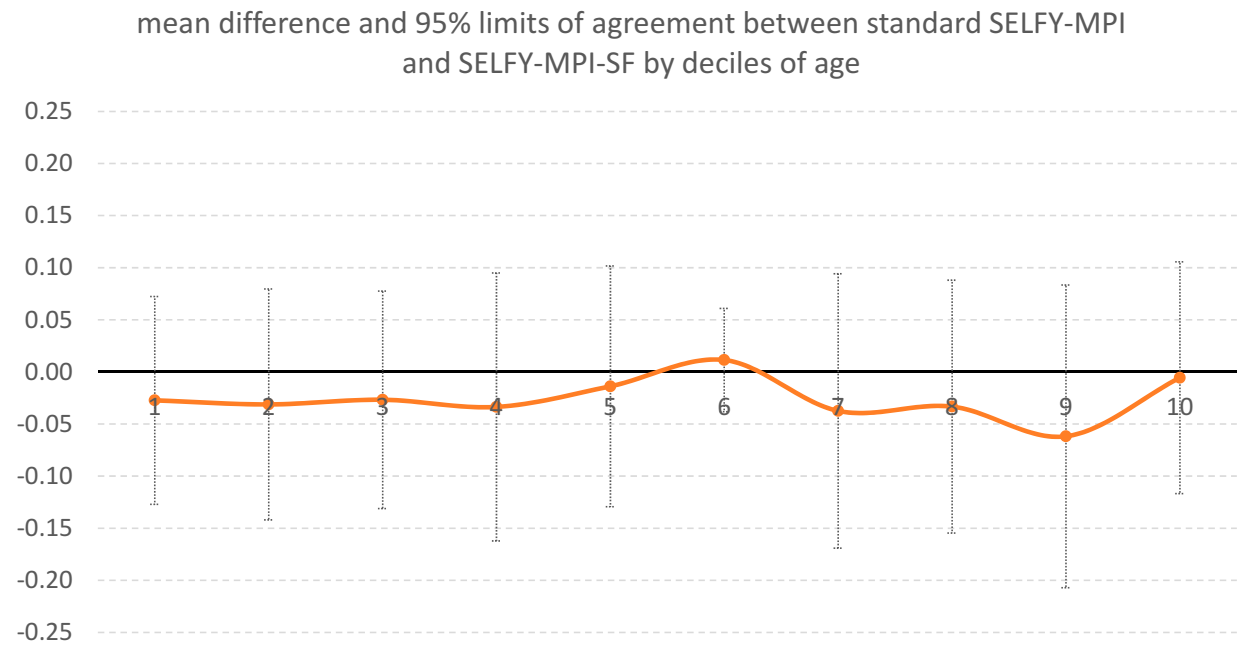

Figure 2 Agreement between standard SELFY-MPI and SELFY-MPI-SF in participants subdivided according to deciles of age. 
a total of 10 self-administered tools for assessing frailty in primary care and concluded that the Tilburg Frailty Indicator and the SHARE Frailty Index were the only suitable ones. ${ }^{9}$ However, as neither of the two was reported to have been compared with a validated scale, their utility in daily clinical practice remains to be confirmed by further research. ${ }^{9}$ The self-administered FRAIL scale recently developed by Morley ${ }^{30}$ is based mainly on the criteria proposed by Fried at al. ${ }^{10}$ and investigates frailty only in terms of physical frailty.

Very recently, a self-administered version of MPI (SELFY-MPI) was developed and validated within the framework of the EFFICHRONIC project, ${ }^{11}$ with the aim of enabling self-assessment of the degree of personal frailty. Like the original MPI, the SELFY-MPI includes not only physical domains but also other important aspects of frailty, ie, comorbidity, polypharmacy, cognition, functional, nutrition and social status. A previous study demonstrated the strong diagnostic agreement between the SELFY-MPI and the MPI, indicating that the SELFYMPI could be used as a predictive tool in communitydwelling subjects. ${ }^{11}$ Like the original MPI, which is able to extract information from CGA to categorize frailty in three subgroups and has excellent prognostic value, ${ }^{31}$ the SELFY-MPI stems from the need for prognostic indices that can be used in the clinical decision-making process in multimorbid and frail older adults, starting from the primary care setting. ${ }^{32}$

Our investigation was aimed at validating a short-form version of the SELFY-MPI (SELFY-MPI-SF), in which the SFES scale has been replaced by the item "cohabitation status"; this item was included in the original version of the MPI in order to underline the relevance of the co-habitation status of the elderly. ${ }^{3,14}$ Our comparison of the two versions of the self-administered MPI showed good agreement between the standard SELFY-MPI and its short-form version (SELFY-MPI-SF), since we observed a very small, and not clinically significant, overestimation in the case of the short-form version (a mean difference of only -0.025 points). Moreover, the BAPs showed that none of the 129 participants fell outside the limits of agreement.

Finally, as expected, we found a highly significant difference in completion time between the two instruments, the average time needed to complete the SFES scale (about 7 mins) being much longer than the $10 \mathrm{~s}$ required, on average, for the simple co-habitation status question. This significant reduction in completion time makes the SELFY-
MPI-SF an even easier self-administered tool for frailty assessment in older people.

The present study has some limitations. First, the cohort enrolled was relatively small, consisting of 129 people enrolled. Second, the study design was crosssectional: longitudinal studies will be required in order to ascertain whether both versions of the SELFY-MPI can predict outcome (mortality or negative outcomes) with the same accuracy as the MPI. Third, as in the case of all selfadministered tools, self-reported data may be influenced by the subject's cognitive status. Moreover, not having more information on the cognitive state of the participants is a further limitation.

\section{Conclusion}

In conclusion, these findings suggest that the new shortform version of the SELFY-MPI (SELFY-MPI-SF) is similar to that of the standard SELFY-MPI, with the advantage that the completion time is shorter. The SELFY-MPI-SF may therefore be an excellent tool for the self-assessment of frailty in community-dwelling older people.

\section{Disclosure}

The authors report no conflicts of interest in this work.

\section{References}

1. Gill TM. The central role of prognosis in clinical decision making. JAMA. 2012;307:199-200.

2. Pilotto A, Panza F. Comprehensive geriatric assessment: evidence. In: Michel JP, Beattie BL, Martin FC, Walston J, editors. Oxford Textbook of Geriatric Medicine. $3^{\text {rd }}$ ed. Chapter 16. Oxford University Press; 2017

3. Pilotto A, Ferrucci L, Franceschi M, et al. Development and validation of a multidimensional prognostic index for one-year mortality from comprehensive geriatric assessment in hospitalized older patients. Rejuvenation Res. 2008;11:151-161. doi:10.1089/rej.2007.0569

4. Yourman LC, Lee SJ, Schonberg MA, et al. Prognostic indices for older adults: a systematic review. JAMA. 2012;307:182-192. doi:10. 1001/jama.2011.1966

5. Pilotto A, Gallina P, Fontana A, et al. Development and validation of a multidimensional prognostic index for mortality based on a standardized multidimensional assessment schedule (MPI-SVaMA) in community-dwelling older subjects. $J$ Am Med Dir Assoc. 2013;14:287-292. doi:10.1016/j.jamda.2013.01.005

6. Pilotto A, Sancarlo D, Polidori MC, et al.; On behalf of the MPI_AGE Investigators. The MPI_AGE European Project: using Multidimensional Prognostic Indices (MPI) to improve cost-effectiveness of interventions in multimorbid frail older persons. Background, aim and design. Eur Geriatr Med. 2015;6(2):184-188. doi:10.1016/j.eurger.2015.01.012.

7. Dent E, Kowal P, Hoogendijk EO. Frailty measurement in research and clinical practice: a review. Eur J Int Med. 2016;31:3-10. doi:10.1016/j.ejim.2016.03.007

8. Dent E, Martin FC, Bergman H, Woo J, Romero-Ortuno R, Walston JD. Management of frailty: opportunities, challenges, and future directions. Lancet. 2019;394(10206):1376-1386. doi:10.1016/ S0140-6736(19)31785-4 
9. Pialoux T, Goyard J, Lesourd B. Screening tools for frailty in primary health care: a systematic review. Geriatr Gerontol Int. 2012;12: 189-197. doi:10.1111/j.1447-0594.2011.00797.x

10. Fried LP, Tangen CM, Walston J, et al. Frailty in older adults: evidence for a phenotype. J Gerontol Biol Sci Med Sci. 2001;56: M146-M156. doi:10.1093/gerona/56.3.M146

11. Pilotto A, Veronese N, Quispe Guerrero KL, et al. Development and validation of a self-administered multidimensional prognostic index to predict negative health outcomes in community-dwelling persons. Rejuvenation Res. 2019;22(4):299-305. doi:10.1089/rej.2018.2103

12. Zora S, Quispe Guerrero KL, Veronese N, et al. Implementation of the SELFY-MPI in five European Countries: a multicenter international feasibility study. Geriatr Care. 2019;5(3):8502. doi:10.4081/ gc. 2019.8502

13. Pimouguet C, Rizzuto D, Lagergren M, Fratiglioni L, Xu W. Living alone and unplanned hospitalizations among older adults: a population-based longitudinal study. Eur J Public Health. 2017;27 (2):251-256. doi:10.1093/eurpub/ckw150

14. Kharicha K, Iliffe S, Harari D, Swift C, Gillmann G, Stuck AE. Health risk appraisal in older people 1: are older people living alone an "at-risk" group? Br J Gen Pract. 2007;57(537):271-276.

15. Tabue Teguo M, Simo-Tabue N, Stoykova R, et al. Feelings of loneliness and living alone as predictors of mortality in the elderly: the PAQUID study. Psychosom Med. 2016;78(8):904-909. doi:10.1097/ PSY.0000000000000386

16. von Elm E, Altman DG, Egger M, et al. The strengthening the reporting of observational studies in epidemiology (STROBE) statement: guidelines for reporting observational studies. $J$ Clin Epidemiol. 2008;61:344-349. doi:10.1016/j.jclinepi.2007.11.008

17. Mahoney FI, Barthel DW. Functional evaluation: the Barthel Index. Md State Med J. 1965;14:61-65.

18. Katz PP; for the Association of Rheumatology Health Professionals Outcomes Measures Task Force. Measures of adult general functional status: the Barthel Index, Katz Index of activities of daily living, health assessment questionnaire (HAQ), MACTAR patient preference disability questionnaire, and modified health assessment questionnaire (MHAQ). Arthritis Care Res. 2003;49(S5):S15-S27. doi:10.10 02/(ISSN)1529-0131

19. Goeppinger J, Doyle M, Murdock B, et al. Self-administered function measures: the impossible dream. Arthritis Rheum. 1985;28:145.

20. Lawton MP, Brody EM. Assessment of older people: self maintaining and instrumental activities of daily living. Gerontologist. 1969;9: 179-186. doi:10.1093/geront/9.3_Part_1.179
21. Brown J, Pengas G, Dawson K, et al. Self administered cognitive screening test (TYM) for detection of alzheimer's disease: cross sectional study. BMJ. 2009;338:b2030. doi:10.1136/bmj.b2030

22. Sancarlo D, D'Onofrio G, Franceschi M, et al. Validation of a modified-multidimensional prognostic index (m-MPI) including the mini nutritional assessment short-form (MNA-SF) for the prediction of one-year mortality in hospitalized elderly patients. $J$ Nutr Health Aging. 2011;15:169-173. doi:10.1007/s12603-010-0293-5

23. Donini LM, Marrocco W, Marocco C, Lenzi A. Validity of the self-mini nutritional assessment (Self-MNA) for the evaluation of nutritional risk. A cross-sectional study conducted in general practice. J Nutr Health Aging. 2018;22:44-52. doi:10.1007/s12603017-0919-y

24. Linn BS, Linn MW, Gurel L. Cumulative illness rating scale. J Am Geriatr Soc. 1968;16:622-626. doi:10.1111/j.1532-5415.1968.tb02103.x

25. Garcia-Caselles P, Miralles R, Arellano M, et al. Validation of a modified version of the Gijon's social familial evaluation scale (SFES): the "Barcelona SFES Version", for patients with cognitive impairment. Arch Gerontol Geriatr Suppl. 2004;9:201-206. doi:10.10 16/j.archger.2004.04.028

26. Garcia Gonzalez JV, Diaz Palacios E, Salamea Garcia A, et al. [An evaluation of the feasibility and validity of a scale of social assessment of the elderly]. Aten Primaria. 1999;23:434-440. Spanish.

27. Fleiss JL. Statistical Methods for Rates and Proportions. 2nd ed. New York: John Wiley \& Sons Inc; 1981.

28. Bland JM, Altman DG. Statistical methods for assessing agreement between two methods of clinical measurement. Lancet. 1986;1:307-310. doi:10.1016/S0140-6736(86)90837-8

29. Abbasi M, Rolfson D, Khera AS, Dabravolskaj J, Dent E, Xia L. Identification and management of frailty in the primary care setting [published correction appears in CMAJ. 2019 Jan 14;191(2):E54]. CMAJ. 2018;190(38):E1134-E1140. doi:10.1503/cmaj.171509

30. Morley JE, Malmstrom TK, Miller DK. A simple frailty questionnaire (FRAIL) predicts outcomes in middle aged African Americans. J Nutr Health Aging. 2012;16:601-608. doi:10.1007/s12603-012-0084-2

31. Committee for Medicinal Products for Human Use (CHMP). Reflection paper on physical frailty: instruments for baseline characterization of older populations in clinical trials. EMA/CHMP/ 778709/2015; 2018

32. Schoenborn NL, Bowman TL II, Cayea D, et al. Primary care practitioners' view on incorporating long-term prognosis in the care of older adults. JAMA Intern Med. 2016;176:671-678. doi:10.1001/ jamainternmed.2016.0670
Clinical Interventions in Aging

\section{Publish your work in this journal}

Clinical Interventions in Aging is an international, peer-reviewed journal focusing on evidence-based reports on the value or lack thereof of treatments intended to prevent or delay the onset of maladaptive correlates of aging in human beings. This journal is indexed on PubMed Central, MedLine, CAS, Scopus and the Elsevier
Bibliographic databases. The manuscript management system is completely online and includes a very quick and fair peer-review system, which is all easy to use. Visit http://www.dovepress.com/ testimonials.php to read real quotes from published authors. 